Ugalde, S. y Pacheco, C. (2021). Restauraciones empíricas en documentos coloniales: identificación de casos en las colecciones del archivo nacional de costa rica. Revista Herencia, Vol. 34 (1), enero-junio, 09-28.

\title{
RESTAURACIONES EMPÍRICAS EN DOCUMENTOS COLONIALES: IDENTIFICACIÓN DE CASOS EN LAS COLECCIONES DEL ARCHIVO NACIONAL DE COSTA RICA
}

\author{
Empirical restorations in colonial documents: identification of cases in the collections of the \\ National Archive of Costa Rica
}

\author{
Sharon Ugalde Soto ${ }^{1}$ \\ St. Jude School, Costa Rica \\ s.ugaldesoto@gmail.com \\ Carlos Pacheco Ureña ${ }^{2}$ \\ Archivo Nacional de Costa Rica, Costa Rica \\ restauracion01@dgan.go.cr
}

Recibido: 20082020

Aprobado: 07092020

1. Bachiller en Antropología y en Bibliotecología; Máster en Dirección de proyectos de conservación y restauración de la Universidad de Barcelona.

Práctica profesional supervisada en el Centro Nacional de Conservación y Restauración de Chile; Departamento de Protección del Patrimonio Cultural del Museo Nacional de Costa Rica; pasantía en Archivo Nacional de Costa Rica; actual coordinadora de la biblioteca de St. Jude School.

2. Pasantía en el Instituto de Conservación y Restauración El Greco de la Universidad Complutense de Madrid.

Artista Creativo Emérito, restaurador de documentos en el Departamento de Conservación del Archivo Nacional de Costa Rica con más de 35 años de experiencia.

\section{RESUMEN}

En las colecciones de documentos coloniales del Archivo Nacional de Costa Rica se encuentran intervenciones que no corresponden a los criterios ni materiales actuales utilizados para la conservación/restauración de papel. El objetivo de este trabajo es investigar dichas restauraciones empíricas, por medio de su identificación y clasificación. Se buscó delimitar además, el periodo en que dichas intervenciones fueron realizadas.

Palabras clave: Restauración de documentos; Documentos coloniales, Intervenciones empíricas; Archivos Nacionales; Costa Rica

\section{ABSTRACT}

In the collections of colonial documents at the National Archive of Costa Rica interventions that do not correspond to the current criteria or materials used for the conservation / restoration of paper have been found. The objective of this paper is to investigate these empirical restorations, by means of their identification and classification. It also seeks to delimit the time period in which these interventions were executed.

Keywords: Documents restoration; Colonial documents; Empirical interventions; National archives; Costa Rica

\section{Introducción}

Al realizar las correspondientes tareas de conservación y restauración de papel en los documentos coloniales que alberga el Archivo Nacional de Costa Rica (ANCR), se han 
encontrado curiosos casos de intervenciones o reparaciones anteriores realizadas sobre los mismos.

La Facultad de Bellas Artes de la Universidad Complutense define las reparaciones como "acciones aplicadas sobre un bien o sobre una parte del mismo para recuperar su funcionalidad y/o su aspecto" (2015). Y según Cortés (2005) y Catalán (2013), la necesidad de arreglar, adobar o reparar objetos deteriorados existe desde siempre, Cortés comenta que "estas prácticas comenzaron siendo ejercidas por los mismos gremios que habían creado las piezas -artesanos y artistas..." (2005, p. 169).

Este trabajo surge del hecho de que estas reparaciones no han sido con anterioridad documentadas ni investigadas. Se considera un aporte valioso además porque al llegar al Departamento de Conservación del Archivo, estos trabajos previos sobre los documentos se eliminan por no cumplir con los estándares actuales de conservación/restauración (Ver: Allo (1997); Vergara (2002); Copedé (2003); Crespo (2007)), por lo que llegará un momento en que todos los documentos habrán sido restaurados y no será posible investigar directamente estas restauraciones empíricas.

La temática de la restauración de papel en Costa Rica ha sido mínimamente estudiada (Cortés, 2002), resulta por tanto fascinante conocer cómo se lidió en el pasado con problemáticas tales como roturas o rasgados, faltantes, ataques biológicos (comején, hongos, etc.) y desprendimientos en los documentos coloniales del país.

\section{Metodología}

La identificación de los casos se realizó por medio de búsquedas aleatorias dentro de las siguientes series coloniales del Archivo:

- Cartago

- Complementario colonial

- Guatemala

- Mortuales coloniales

- Mortuales independientes: Cartago, Puntarenas y Guanacaste

- Protocolos coloniales 
Estos documentos se encuentran agrupados en contenedores de cartón (la cantidad de expedientes por caja depende de los volúmenes). Se tomaron cajas aleatoriamente y se revisaron todos los documentos. Por serie se examinaron de 15 a 20 cajas.

Para poder entender y ordenar los casos encontrados, se realizó una clasificación de las intervenciones según su propósito: 1) soportes para la encuadernación, 2) unión de rasgados mediantes productos adhesivos 3) parches-injertos, 4) laminaciones y 5) protección de caracteres externos.

En cuanto a la identificación de la época en que fueron realizadas las reparaciones, se siguieron dos líneas de investigación para delimitar un posible rango de tiempo; en primer lugar, se realizaron entrevistas a personas exfuncionarias clave del Archivo y en segundo, se llevó a cabo una búsqueda bibliográfica dentro de la institución con el fin de encontrar referencias o documentación sobre las intervenciones halladas.

\section{Las colecciones de documentos coloniales del Archivo Nacional}

En una publicación del Archivo Nacional (1981) se comenta que las autoridades españolas del naciente imperio se interesaron desde años tan tempranos como 15301548 en conservar documentación oficial tal como cédulas o provisiones en favor de ciudades y entidades administrativas. En esa época se establecieron normas para que

\footnotetext{
"ciudades y villas por igual guardasen los papeles más importantes, en un archivo, o arca de tres llaves, en manos de tres sujetos diferentes y relevantes para la comunidad..." (p. 6).
}

A pesar de este esfuerzo, no siempre fue posible conservar estos papeles adecuadamente, ya fuese por las vicisitudes mismas de las ciudades, por las condiciones medioambientales presentes o por la calidad de los papeles y las tintas. Recalca esta publicación que debido a estos factores, el Archivo resguarda en realidad pocos documentos del siglo XVI. Aparte, Murillo (1980) menciona como causas de perdida de documentos coloniales en la época, el saqueo de piratas y la sustracción. 
Aparte, en 1882, el gobierno de Guatemala donó la documentación judicial producida en nuestro país que había sido enviada en tiempos coloniales a la Real Audiencia para su sentencia definitiva (Archivo Nacional, 1981). Los documentos coloniales conservados en el Archivo Histórico del ANCR se dividen de la siguiente manera:

Cuadro 1. Descripción de las colecciones coloniales del ANCR.

\begin{tabular}{|c|c|c|c|}
\hline $\begin{array}{c}\text { Nombre de la } \\
\text { colección o fondo }\end{array}$ & $\begin{array}{c}\text { Años } \\
\text { abarcados }\end{array}$ & Descripción de los documentos & Cantidad \\
\hline Cartago & $1578-1830$ & $\begin{array}{l}\text { Documentación producida en la antigua capital } \\
\text { de Costa Rica durante el período colonial. Incluye } \\
\text { asuntos propios de la gobernación, bandos, } \\
\text { reales cédulas y provisiones; así mismo, } \\
\text { licitaciones, cartas, expedientes, padrones, } \\
\text { órdenes de pago, mandatos, actas, } \\
\text { certificaciones, reales decretos y } \\
\text { correspondencia. }\end{array}$ & $\begin{array}{l}1150 \\
\text { document } \\
\text { os } \\
\text { textuales }\end{array}$ \\
\hline $\begin{array}{l}\text { Complementario } \\
\text { colonial }\end{array}$ & $1517-1821$ & $\begin{array}{l}\text { Producto de gestiones, trámites, solicitudes y } \\
\text { resoluciones de los órganos administrativos, } \\
\text { hacendarios, judiciales y particulares. Compuesto } \\
\text { por los siguientes tipos documentales: reales } \\
\text { cédulas y provisiones, informes, bandos, autos, } \\
\text { causas criminales, cartas, listas, expedientes, } \\
\text { estados de nacimientos, libros de cuentas, etc. }\end{array}$ & $\begin{array}{l}7.501 \\
\text { unidades } \\
\text { document } \\
\text { ales }\end{array}$ \\
\hline Guatemala & $1539-1821$ & $\begin{array}{l}\text { Informes de gobernadores, obispos y misioneros } \\
\text { (y sobre causas criminales), padrones de pueblos, } \\
\text { cuentas, testimonios, sumarias, testimonios de } \\
\text { sucesión, litigios, juicios de residencia, reclamos } \\
\text { de impuestos, informaciones de méritos y } \\
\text { servicios, reclamos eclesiásticos y de dinero, } \\
\text { causas criminales, pedimentos, testamentos, } \\
\text { consultas, demandas, reales provisiones y } \\
\text { cédulas, peticiones, cartas, etc. }\end{array}$ & $\begin{array}{l}1181 \\
\text { unidades } \\
\text { document } \\
\text { ales }\end{array}$ \\
\hline $\begin{array}{l}\text { Mortuales } \\
\text { coloniales }\end{array}$ & $1641-1830$ & $\begin{array}{l}\text { Inventarios de bienes, así como listas de nombres } \\
\text { de personas unidas históricamente por vínculos } \\
\text { familiares. Predominan expedientes de juicios de } \\
\text { sucesión de bienes. }\end{array}$ & $\begin{array}{l}2485 \\
\text { unidades } \\
\text { document } \\
\text { ales }\end{array}$ \\
\hline Protocolos & $1602-1961$ & Protocolos de escribanos (coloniales) & $\begin{array}{l}\text { Sin } \\
\text { especificar }\end{array}$ \\
\hline
\end{tabular}

Cuadro elaborado con información obtenida de Dirección General del Archivo Nacional (2018) 


\section{Tipos de intervenciones identificadas: técnicas y materiales}

Se documentaron un total de 30 expedientes con reparaciones. Estas se clasificaron según su propósito en el documento, idea basada en la clasificación presentada por Dávila (2013) de reparaciones en cerámica arqueológica; la autora identifica restauraciones antiguas en vasijas cerámicas, realizando una clasificación tipológica a partir del material conocido y recopilado en la bibliografía.

Debido a que el tema de reparaciones o restauraciones antiguas ha sido poco tratado no solo a nivel de cerámica, sino a nivel general en las disciplinas de la arqueología y la restauración (Davila, 2013, p. 453), el enfoque utilizado por la autora sirvió como base para realizar la clasificación propia, adaptada al material papel y al tipo de intervenciones encontradas.

Cuadro 2. Expedientes coloniales organizados según las reparaciones identificadas.

\begin{tabular}{|c|c|c|c|}
\hline $\begin{array}{l}\text { Tipo de reparación } \\
\text { identificada }\end{array}$ & Fondo o colección & Expedientes & Materiales empleados \\
\hline \multirow[t]{2}{*}{$\begin{array}{l}\text { Uso de escartivanas } \\
\text { para la } \\
\text { encuadernación }\end{array}$} & $\begin{array}{l}\text { Complementario } \\
\text { colonial }\end{array}$ & $\begin{array}{ll}1086, & 3295, \\
3387, & 3444, \\
3462, & 3467, \\
3468, & 3873, \\
5485 & \end{array}$ & \multirow[t]{2}{*}{$\begin{array}{l}\text { Diferentes tipos de papeles } \\
\text { empleados como escartivanas } \\
\text { (papel antiguo de algodón y lino, } \\
\text { papel de pasta mecánica, papel } \\
\text { glassine y adhesivo), encolante } \\
\text { (origen desconocido) }\end{array}$} \\
\hline & $\begin{array}{l}\text { Protocolos } \\
\text { coloniales }\end{array}$ & 1239 & \\
\hline \multirow{3}{*}{$\begin{array}{l}\text { Unión de rasgados } \\
\text { mediante productos } \\
\text { adhesivos }\end{array}$} & $\begin{array}{l}\text { Protocolos } \\
\text { coloniales }\end{array}$ & 1039 & \multirow{3}{*}{$\begin{array}{l}\text { Diferentes tipos de papeles } \\
\text { empleados para unir rasgados } \\
\text { (papel de pasta mecánica, papel } \\
\text { glassine y adhesivo), encolante } \\
\text { (origen desconocido) }\end{array}$} \\
\hline & $\begin{array}{l}\text { Complementario } \\
\text { colonial }\end{array}$ & 3444 & \\
\hline & Cartago & 1085,1093 & \\
\hline \multirow[t]{2}{*}{ Parches - injertos } & $\begin{array}{l}\text { Complementario } \\
\text { colonial }\end{array}$ & $\begin{array}{ll}3311, & 3392, \\
3398, & 3444, \\
3457, & 3462, \\
5449 & \end{array}$ & \multirow{2}{*}{$\begin{array}{l}\text { Diferentes tipos de papeles } \\
\text { empleados para hacer injertos } \\
\text { (papel de pasta mecánica, papel } \\
\text { glassine y adhesivo), encolante } \\
\text { (origen desconocido) }\end{array}$} \\
\hline & $\begin{array}{l}\text { Mortuales } \\
\text { independientes } \\
\text { Puntarenas }\end{array}$ & 1 & \\
\hline
\end{tabular}




\begin{tabular}{|c|c|c|c|}
\hline & Cartago & $1,25,1092$ & \\
\hline \multirow[t]{3}{*}{ Laminaciones } & $\begin{array}{l}\text { Complementario } \\
\text { colonial }\end{array}$ & 3309 & \multirow{3}{*}{$\begin{array}{l}\text { Diferentes tipos de papeles } \\
\text { empleados para reforzar y } \\
\text { reintegrar hojas (papel antiguo } \\
\text { de algodón y lino, papel de pasta } \\
\text { mecánica y papel glassine), } \\
\text { encolante (origen desconocido) }\end{array}$} \\
\hline & $\begin{array}{l}\text { Protocolos } \\
\text { coloniales }\end{array}$ & 1040 & \\
\hline & Cartago & 1085 & \\
\hline $\begin{array}{l}\text { Protección de } \\
\text { caracteres externos }\end{array}$ & Cartago & $\begin{array}{l}1086, \quad 1091, \\
1092,1093\end{array}$ & $\begin{array}{l}\text { Papel antiguo de algodón y lino, } \\
\text { encolante (origen desconocido), } \\
\text { cera (origen desconocido) }\end{array}$ \\
\hline
\end{tabular}

Cuadro de elaboración propia a partir de las colecciones del Archivo Nacional de Costa Rica mencionadas en el apartado de metodología

\section{Uso de escartivanas para la encuadernación}

La escartivana es una tira de papel o en ocasiones de tela que "se pone en las láminas u hojas sueltas para que se puedan encuadernar de modo conveniente" (Real Academia Española, 2019). En los casos encontrados en el Archivo, era utilizada como un medio para evitar perforar los folios de los expedientes al realizar las encuadernaciones.

Esta modificación a los documentos originales se encontró en todos los ejemplares revisados compuestos por más de una hoja, pero no se empleó de modo uniforme; fueron utilizados diferentes tipos de papeles para cumplir con la función de escartivanas. Se identificaron tres tipos de papel: papel glassine, un papel de pasta mecánica (Figura 1) y papel antiguo de algodón y lino, el mismo utilizado para los documentos.

Figura 1. Papel de pasta mecánica empleado como escartivana, expediente 3467.

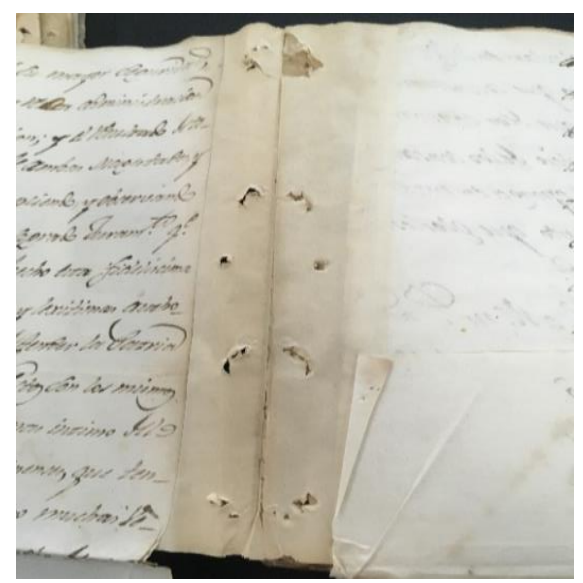

Autoría propia 
Las escartivanas fueron unidas a los documentos con un encolante no identificado, algunos folios presentan un uso excesivo del encolante (Figura 2) lo que causó que el mismo se transfiriera a otros folios. Además en algunos casos se emplearon dos o los tres tipos de papel como escartivana en un mismo expediente (ejemplos: 3873, 3444, 3467 y 3468$)$.

Por tanto, la escogencia de los papeles a utilizar parece deberse a una cuestión de disponibilidad, más que de estética. Relacionado con esto, no se observó en los casos identificados una intención de esconder o disimular las reparaciones, práctica existente a mediados del siglo veinte (Schweidler, 2006).

Figura 2. Exceso de cola aplicada en la escartivana, expediente 5485.

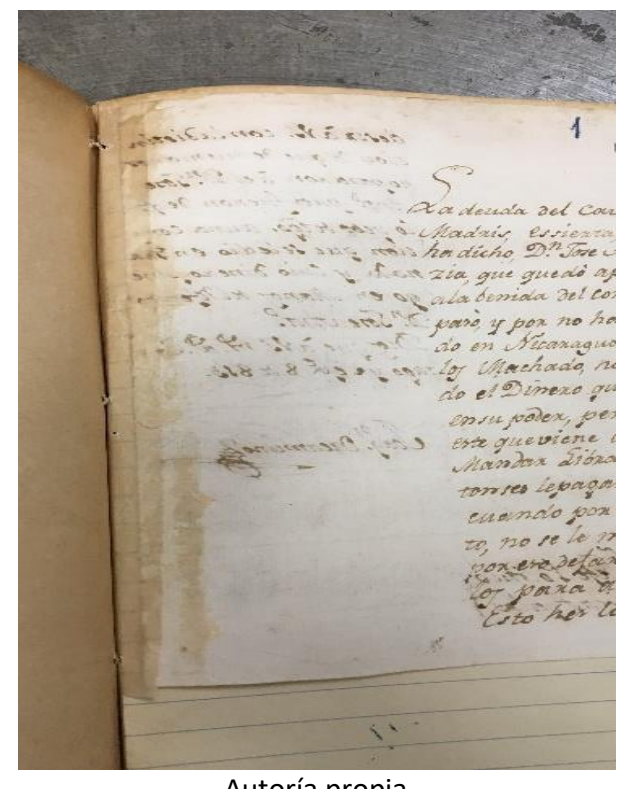

Autoría propia

\section{Unión de rasgados mediante productos adhesivos}

El rasgado corresponde a una rotura producida en el tejido del papel (Calvo, 1997). Los desgarros se unen con el fin de reforzar el papel y evitar que el rasgado se haga más grande y se desprenda una parte del documento, el cual parece ser el fin de estas reparaciones empíricas. Dentro de los expedientes revisados se encontraron uniones de 
rasgados utilizando papel de pasta mecánica, papel glassine y papel adhesivo; estos fueron colocados sobre los rasgados con encolante.

A diferencia del criterio actual, tanto funcional como estético de cubrir solamente la sección en donde se encuentra el rasgado, se puede observar en varios casos identificados el uso excesivo de papel en relación con el tamaño de los rasgados (Figura 3).

Figura 3. Unión de rasgados con papel de pasta mecánica y encolante, expediente 1085.

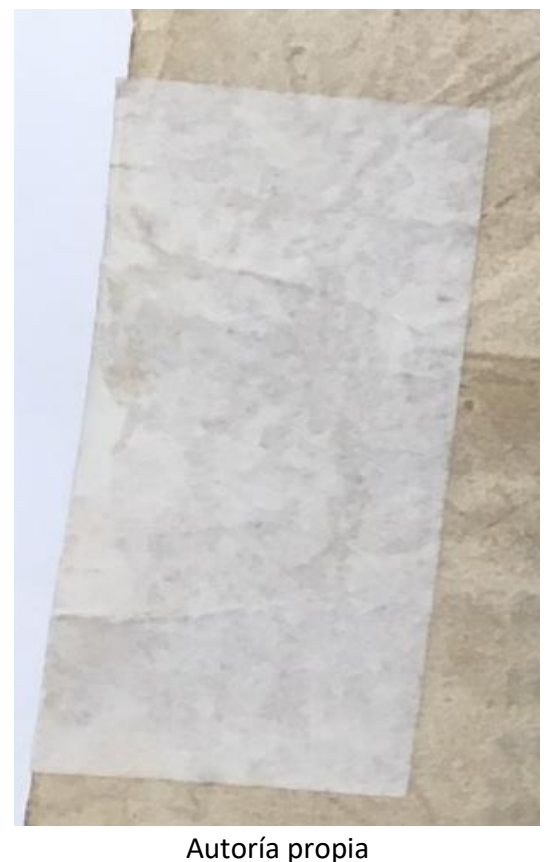

En la figura 4, se puede observar un caso en donde no solo se cubre una sección de papel sin rasgado, sino que para reparar un rasgado largo horizontal, se llega a sobreponer el papel sobre el texto del documento. Actualmente en una intervención de este tipo no se cubriría una sección tan grande del folio, solamente lo mínimo para obtener un mejor acabado. Es importante resaltar además que utilizar franjas de papel tan grandes y recortadas sin dejar fibras libres que se unan al documento, causa en el folio arrugamientos y deformaciones (Figuras 3 y 4 ). 
Figura 4. Rasgado horizontal, unido con papel glassine y adhesivo, expediente 3444.

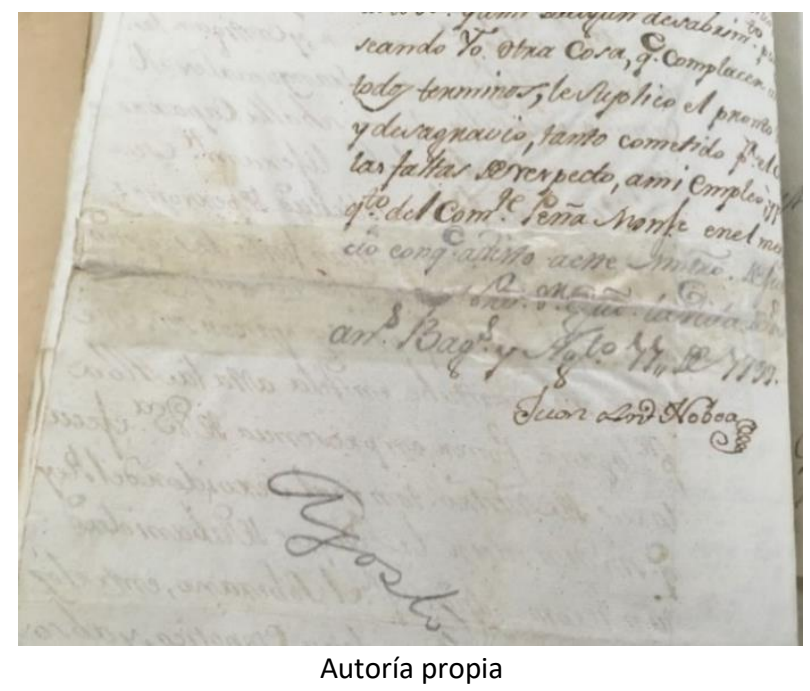

\section{Parches - injertos}

Calvo define el injerto como "la pieza colocada en una laguna del soporte de una obra..." (1997, p. 121). Y les diferencia de los parches en que los injertos se limitan al hueco exacto de la laguna. En los casos analizados se puede hablar de parches utilizados con el fin de cumplir la función de un injerto.

Otra característica que los diferencia de los injertos actuales, es que fueron realizados en casi la totalidad de los casos en un solo lado del folio y se utilizaron diferentes tipos de papeles como parches: papel de pasta mecánica, papel glassine y papel adhesivo así como encolante (no identificado); y en esta categoría tampoco se presenta un criterio de uniformidad, se encontraron expedientes con varios parches, hechos con diferentes papeles (expedientes 1092, 3311 y 3457).

Si bien estas intervenciones no calzan como injertos ya que no cumplen con el requisito de cubrir el espacio exacto del faltante, en todos los casos hallados se respetó el borde de la hoja. En algunos ejemplos se visualizó un uso excesivo de encolante (Figura 5), lo que causa que el folio se vea manchado.

En el caso de los parches colocados con papel adhesivo (Figura 6), es posible observar deformaciones importantes debido a la contracción del material. Otro punto a 
mencionar es que no se tuvo el cuidado de estirar pequeños dobleces para facilitar su apreciación y lectura (Figura 5).

Figura 5. Parche de papel de pasta mecánica, con exceso de encolante y dobleces, expediente 3392.

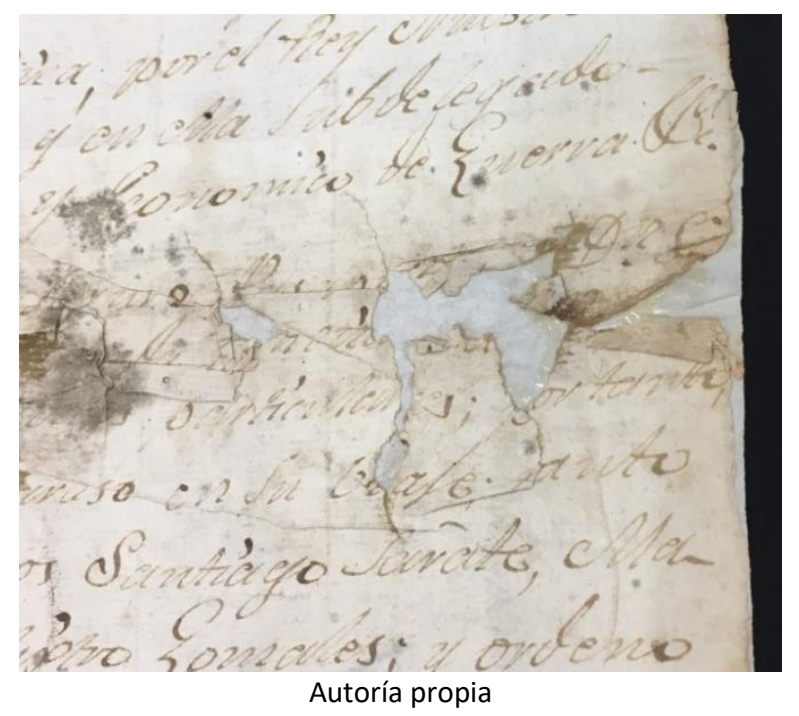

Figura 6. Parche de papel adhesivo, expediente 1.

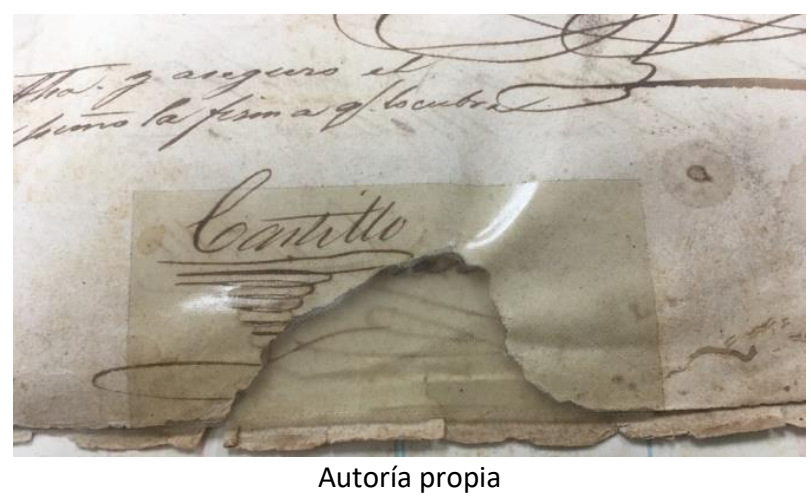

Además, se puede observar el uso de parches para contrarrestar los efectos causados por dos tipos de ataques biológicos, en la figura 7 se utilizó un parche de glassine para cubrir un faltante causado por comején y en la figura 8, se utilizó el papel glassine como parche para darle estabilidad a un documento fuertemente afectado por un hongo.

Figura 7. Parche con papel glassine, faltante causado por comején, expediente 3444. 


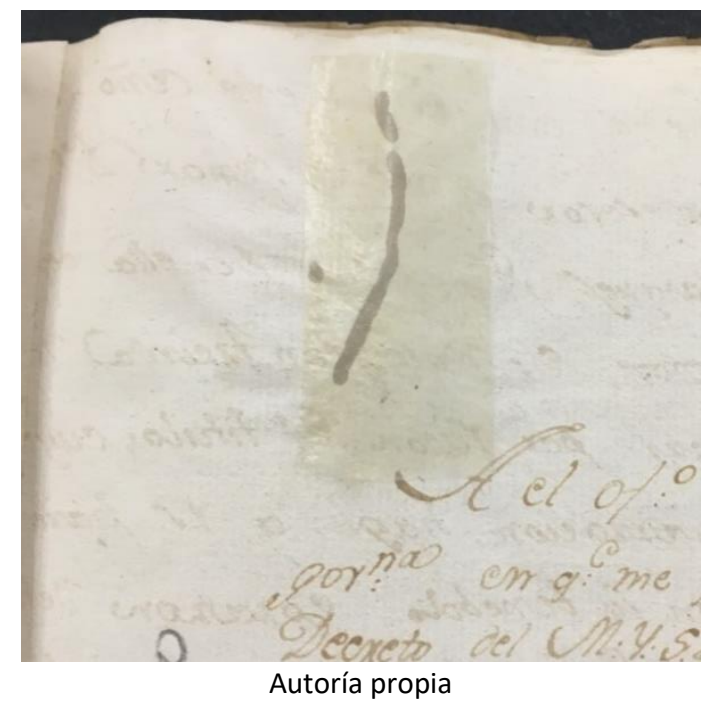

Figura 8. Parche con papel glassine, faltante causado por hongo, expediente 3462.

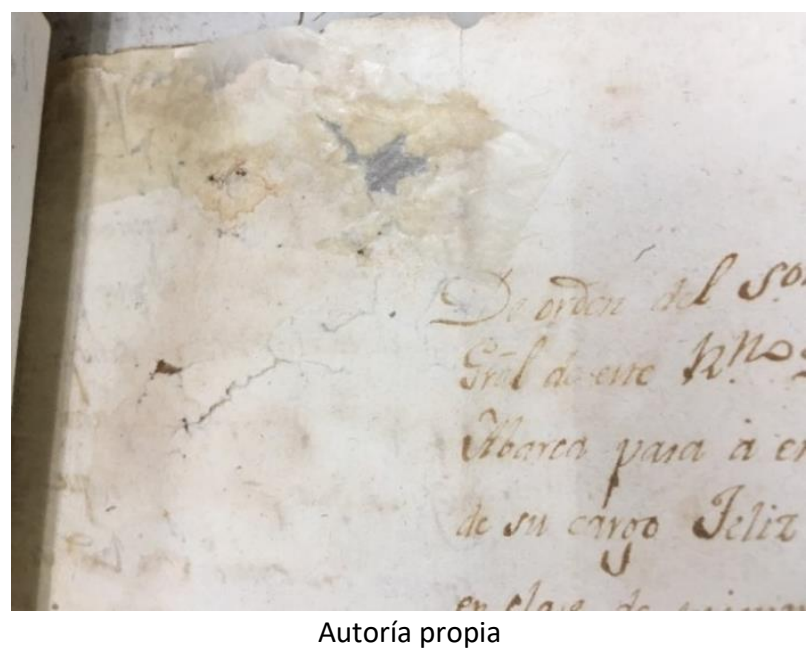

\section{Laminaciones}

Se encontraron dos casos muy diferentes entre sí pero que tienen una característica en común, en ambos se utilizó el material de laminación para crear injertos en áreas con faltantes importantes. La laminación se define como una técnica de la restauración de papel que:

"consiste en adherir a una o ambas caras de un documento un refuerzo que proporciona la consistencia necesaria para su mejor manejo y conservación" (Calvo, 1997, p. 130).

El primer caso corresponde a un expediente de dos folios (Figura 9), ambos con lagunas muy grandes, y laminados por un solo lado. Curiosamente, a pesar de ser un documento 
con solo dos folios se decidió laminarlos con papeles distintos, uno con papel glassine y el otro con un papel de pasta mecánica. En los dos folios es visible el exceso de encolante empleado.

Figura 9. Laminación con papel glassine y papel de pasta mecánica por un solo lado, expediente 3309.

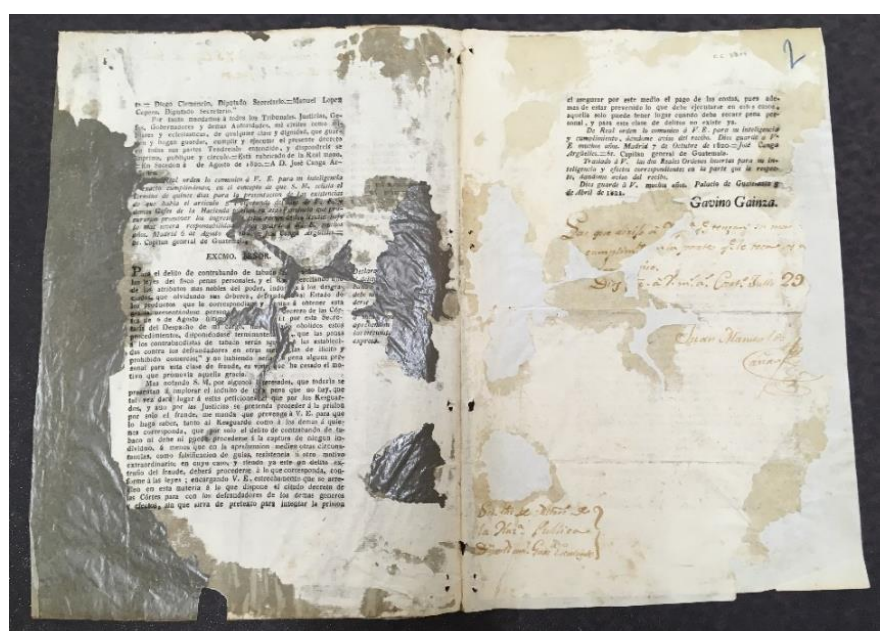

Autoría propia

El siguiente caso corresponde a una laminación parcial (Figura 10). Se laminó solamente la mitad inferior del folio, pero a diferencia del caso anterior, la laminación se hizo por ambos lados. El tipo de papel, glassine, dificulta la lectura del documento.

Figura 10. Laminación parcial con papel glassine por ambos lados, expediente 5449.

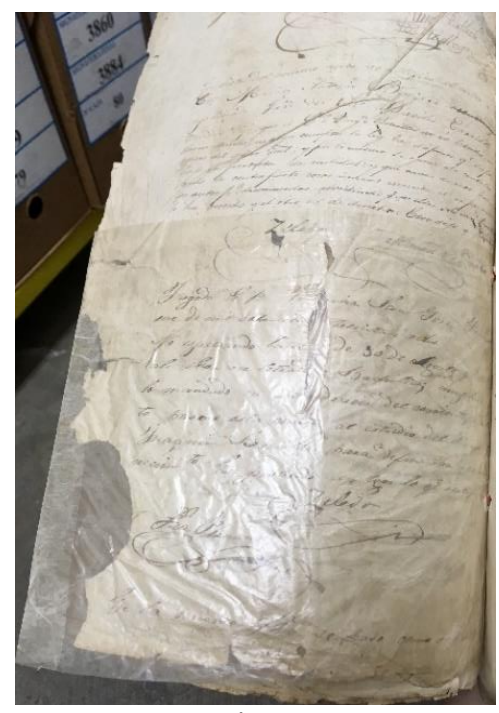

Autoría propia 


\section{Protección de caracteres externos}

Los caracteres externos son en la gestión documental, los elementos "que configuran la apariencia externa de los documentos" (Taedium, s.f.), como por ejemplo las tintas, signos especiales, sellos, etc. En los expedientes revisados se encontraron sellos de cera protegidos con un papel cortado del tamaño del sello, el cual a su vez tiene cuatro pestañas para brindarle más protección y decoración (Figura 11). El papel utilizado es el mismo de los documentos, de lino y algodón.

Figura 11. Sistema de protección en papel para sello, expediente 1086.

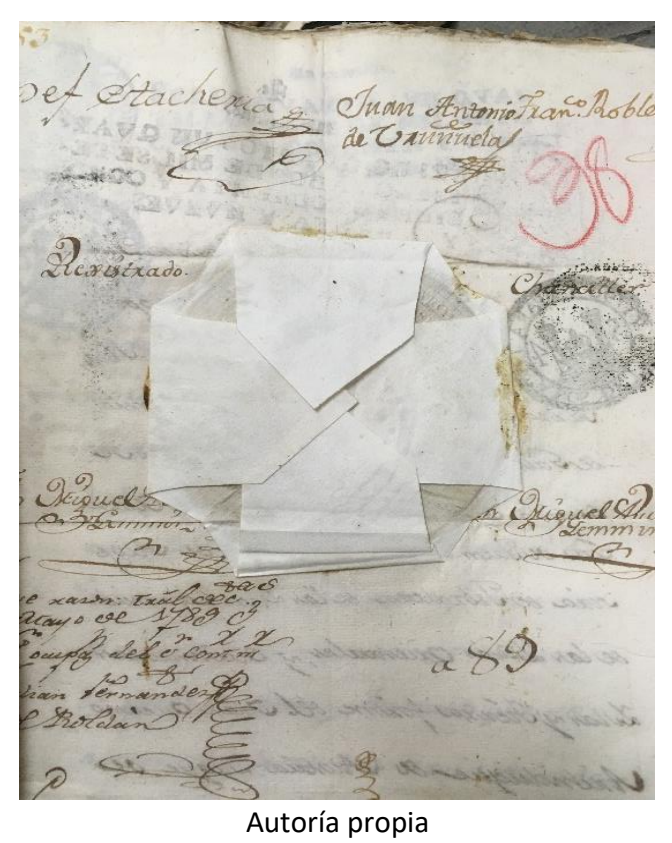

Los sellos han ido perdiendo su agarre en los folios, pero el sistema de protección colocado sobre ellos permite que no se desprendan. La parte posterior de los folios en donde hay un sello se han manchado, pero debido a las viñetas (Figura 12), las hojas anteriores al sello no se han visto afectadas.

Esta medida de protección de los sellos es la única que se puede fechar con exactitud ya que se hizo al momento de elaborar los documentos. Los cuatro casos hallados pertenecen a la colección colonial Cartago $(1086,1091,1092,1093)$ y datan de los años $1793-1821$. 
Figura 12. Sistema de protección con pestañas abiertas, expediente 1086.

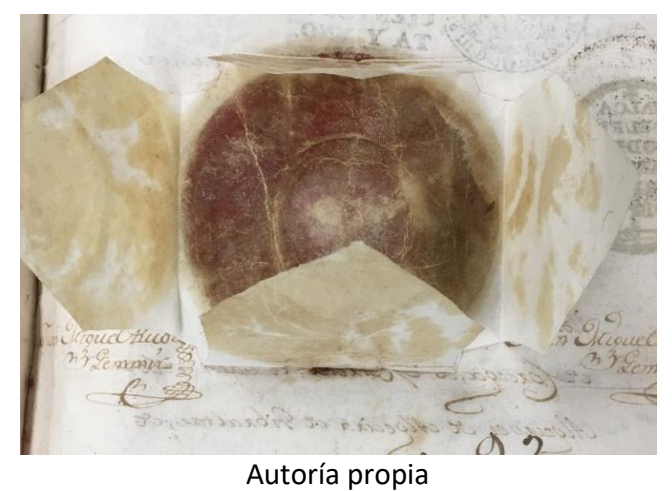

\section{Posibles indicios de la época en que fueron realizadas las reparaciones encontradas en los documentos coloniales}

La primera referencia sobre reparaciones hechas a los documentos coloniales del Archivo Nacional data de 1885, la misma corresponde a un informe de labores por parte del entonces director del Archivo, Francisco María Iglesias Llorente (director entre los años 1883-1890) (Murillo, 1980).

Iglesias comenta que encontró los documentos en un estado de total abandono, inclusive dice que muchos de los documentos se encontraban en el suelo del Palacio Nacional; y que por tanto, él y los otros dos funcionarios que tenía la institución en ese momento tuvieron que darse a la tarea de "sacudir, desarrugar, remendar, coser o embrochar, tantos papeles, coordinándolos provisionalmente a fin de simplificar el ulterior trabajo" (Iglesias, 1885, p. 313).

Menciona que los documentos se encontraban almacenados con tablillas y ataduras que los estrujaban y no permitían que fueran consultados, por lo que tomaron la decisión de soltarlos. Inclusive detalla cuales series y cuantos documentos fueron arreglados y cosidos o forrados. Las series con documentos coloniales que intervino Iglesias son:

- Cartago, 250 ejemplares impresos

- Juicios civiles, 1382 expedientes

- Causas criminales, 614 expedientes

- Expedientes gubernativos, 1621 expedientes

- Causas mortuales, 939 expedientes (Iglesias, 1885). 
El informe de Iglesias es bastante detallado, lo cual corresponde a una excepción con respecto a los otros informes de la época. Los otros reportes, incluyendo otros del mismo Iglesias, no sobrepasan un par de páginas; se revisaron los informes de 1881 a 1916 compilados por Jaén (2017), así como correspondencia y otros informes de labores del Archivo hasta el año 1980.

Estos otros reportes tampoco aluden nada con respecto a reparaciones, remiendos o restauraciones a los documentos. El tema de las reparaciones o remiendos no se vuelve a mencionar; pero a partir de 1904 se empieza a hablar en los informes de un pequeño departamento de encuadernación (compuesto por dos personas) (Jaén, 2017).

Hasta el año 1973 se empieza a discutir en la institución sobre la necesidad de espacios, equipo y formación para los procesos de conservación y reproducción de documentos (Murillo, 1980, p. 78). Esto es respaldado por dos entrevistas realizadas a funcionarios de esta época, se entrevistó a Luz Alba Chacón León (funcionaria del Archivo desde 19581991 y Directora del mismo de 1980-1991) y a Rodrigo Morera Herrera (funcionario del Archivo y del Departamento de Conservación desde 1974-2006).

De acuerdo con la primera, cuando ingresó al Archivo solamente existía una sección de encuadernación. Y según el señor Morera, cuando el ingresó al Archivo, había además una sección de microfilmación activa. En 1975, el coordinador de dicha sección, Emilio Chávez Guzmán es enviado a España para especializarse en restauración de documentos y el señor Morera viaja a República Dominicana en 1976 para formarse en restauración y microfilmación, por lo que en los años posteriores se consolidan ambas áreas.

Las entrevistas arrojaron dos datos muy valiosos. En primer lugar, que posiblemente las reparaciones son anteriores a 1958 ya que en dicho momento en el Archivo no se realizaban procedimientos de restauración según el criterio de los entrevistados, solamente de encuadernación y microfilmación; en segundo, que a partir de 1975, durante las estancias de formación en el extranjero, se aprendieron y empezaron a aplicar en el Archivo técnicas y criterios de conservación y restauración muy similares a 
los que se utilizan actualmente, utilizando por ejemplo carboximetil celulosa para la unión de rasgados o hacer injertos con papel japonés (L. A. Chacón León, comunicación personal, 26 de febrero del 2019 y R. Morera Herrera, comunicación personal, 27 de marzo del 2019).

Establecer los años en que se realizaron las intervenciones encontradas en los documentos coloniales es tarea casi imposible por la falta de información; ya que se reporta que los documentos que iniciaron los Archivos Nacionales se encontraban en un estado de deterioro y abandono importante (Iglesias, 1885), tiene sentido pensar que no fue antes de la creación de esta institución cuando se intervinieron, pero al no haber información sobre como fue el manejo de estos documentos en la época colonial, no se podría realizar una afirmación al respecto.

Tanto la cantidad como el volumen de los reportes o informes de labores en los inicios del Archivo Nacional son escasos y muy generales. La única mención directa a reparaciones o remiendos es hecha por Francisco Iglesias en 1885, pero tampoco se menciona específicamente que tipo de remiendos o con que materiales se hicieron, por tanto no podemos tampoco dar por un hecho que fue él, ni en este periodo en que se realizaron las intervenciones.

Otro punto importante a destacar es que si bien no existió como una sección dentro del organigrama del Archivo hasta 1982 (Chacón y Chacón, 1991), desde 1904 se habla en informes de un pequeño departamento de encuadernación dentro de la institución. Una opción muy posible es que al hacer las encuadernaciones en este departamento se hicieran las reparaciones y que estas intervenciones simplemente no se reportaran, ya que se consideraban parte de la encuadernación.

\section{Conclusiones}

Por medio de la clasificación de los casos encontrados, fue posible ordenar estas reparaciones empíricas y entenderlas en función de sus propósitos en los documentos. A pesar de que los materiales y las técnicas empleadas en las intervenciones 
documentadas no sean las que se utilizarían hoy día, tanto por criterios estéticos como funcionales y de idoneidad de materiales, es claro que las reparaciones se hicieron pensando en alargar la vida de los documentos y generarles estabilidad o firmeza para que pudieran ser consultados posteriormente.

Y a pesar de no contar con los conocimientos de conservación/restauración, las ideas en sí no son tan distintas a las practicadas actualmente, lo que cambia es la ejecución. El uso de escartivanas para encuadernar, la unión de rasgados, los injertos, las laminaciones y el uso de elementos extra para proteger caracteres externos, son todos métodos que utiliza actualmente el profesional en conservación para proteger un documento.

En cuanto a la fecha de realización de las alteraciones, si bien no es posible establecer exactamente cuándo se iniciaron los procesos de reparación empírica de los documentos (excepto para el tipo Protección de caracteres externos); gracias a las referencias consultadas y a las entrevistas, si se pudo establecer que las intervenciones se hicieron antes de 1975, año en que los funcionarios de la sección de encuadernación y posterior departamento de conservación empezaron a formarse en el exterior con las técnicas, materiales y procedimientos profesionales de la restauración de documentos.

Surgen nuevos temas que se pueden estudiar a partir de este primer acercamiento. Por un lado, se podría investigar el origen de los papeles y cintas adhesivas empleadas en los diferentes tipos de intervenciones para delimitar un poco más cuando se realizaron estas. Se genera también la interrogante de si estas intervenciones anteriores deberían ser eliminadas, ya sea en su totalidad, parcialmente o si deberían mantenerse algunos ejemplos (Catalán, 2013), pensando en que forman parte de la historia de los documentos.

Nace también la pregunta: ¿cuáles son algunos de los materiales que se emplearon en estas reparaciones y si estos están afectando negativamente la preservación de los expedientes? Por ejemplo, no se encontraron referencias sobre los tipos de encolantes 
empleados en las intervenciones. Utilizando métodos científicos de análisis como la Espectroscopia de Transmisión de Infrarrojo con Transformada de Fourier (FTIR) sería posible conocer más a profundidad estos materiales y por ende, determinar si los mismos podrían causar un daño importante o irreversible a los documentos.

Con esta idea en mente, al documentar los casos para este artículo se tomaron a su vez muestras de diferentes elementos de interés (encolantes, cintas adhesivas, papeles, ceras) con el fin de analizarlas y presentarlas en una investigación que se está realizando en conjunto con personal del Centro de Investigación en Ciencia e Ingeniería de Materiales (CICIMA) de la Escuela de Química de la UCR.

\section{Agradecimientos}

Al personal del Archivo Nacional que nos brindó su ayuda en la búsqueda de documentos y recomendaciones para el artículo; una mención especial al Jefe del Departamento de Conservación, el señor Marco Calderón Delgado por todo su apoyo con la realización del trabajo y al señor Javier Gómez Jiménez, Jefe del Departamento de Archivo Histórico, por facilitarnos la búsqueda dentro de los archivos.

También a la señora Luz Alba Chacón León y al señor Rodrigo Morera Herrera por permitirnos entrevistarlos. Por último, un gran agradecimiento a la doctora Mavis Montero Villalobos de la Escuela de Química de la Universidad de Costa Rica por la valiosa retroalimentación y por el gran interés de realizar trabajo interdisciplinario en el Archivo; esperamos a partir de este trabajo, generar nuevos cuestionamientos.

\section{Bibliografía}

Allo Manero, A. (1997). Teoría e historia de la conservación y restauración de documentos. Revista general de documentación e información, 7(1), 253295. https://revistas.ucm.es/index.php/RGID/article/viewFile/RGID9797120253A/11042

Archivo Nacional de Costa Rica (1981). Centenario: 1881-1981. San José, Costa Rica: Ministerio de Cultura, Juventud y Deportes. 
Calvo, A. (1997). Conservación y restauración: materiales, técnicas y procedimientos de la A a la Z. Barcelona, España: Eds. del Serbal.

Catalán Mezquíriz, E. (2013). Evolución de criterios en la conservación y restauración de cerámicas: intervenciones antiguas versus nuevas intervenciones. Anales del Museo de América, (XXI), 241-251. https://dialnet.unirioja.es/descarga/articulo/4831379.pdf

Chacón León, L. A. (1986). El departamento de conservación del Archivo Nacional. Archivese, (7), 1.

Chacón Arias, V. y Chacón León, L. A. (1991). El Archivo Nacional de Costa Rica. Cuadernillos del Archivo Nacional, (1), 1-2.

Copedé, M. (2003). Restauración del papel: prevención, conservación, reintegración. España: Ed. Nerea.

Cortés Arjona, J. (2005). Acercamiento a la restauración de bienes bibliográficos. En: I Seminario sobre Patrimonio Bibliográfico Vasco. Fundación Sancho El Sabio, VitoriaGasteiz. https://dialnet.unirioja.es/servlet/articulo?codigo=4142455

Cortés Ramírez, W. (2002). Informe final de investigación: restauración de documentos textuales en soporte de papel en Costa Rica. repositorio.sibdi.ucr.ac.cr:8080/jspui/bitstream/123456789/360/1/22798.pdf

Crespo Arcá, L. (2007). Los fondos de los archivos: el equilibrio entre su uso y los criterios de restauración. En: II Jornadas Técnicas sobre Restauración de Documentos: Criterios de intervención en la restauración de libros y documentos. Archivo Real y General de Navarra.

www.mcu.es/ccbae/es/catalogo_imagenes/imagen.cmd?path=1009\&posicion=1

Dávila, C. (2013). Evidencias arqueológicas de restauración de cerámica. Técnicas antiguas de reparación y recuperación de uso. En: Monografías ex officina hispana 1, 453-473.

https://www.researchgate.net/publication/256979190_Evidencias_arqueologicas_de_re stauracion_de_ceramica_Tecnicas_antiguas_de_reparacion_y_recuperacion_de_uso

Dirección General del Archivo Nacional (2018). Guía de información custodiada en el Departamento de Archivo Histórico. http://www.archivonacional.go.cr/index.php?option=com_content\&view=article\&id=21 7:guia-de-fondos\&catid=69:archivo-historico\&ltemid=91 
Facultad de Bellas Artes de la Universidad Complutense de Madrid (2015). Terminología básica de conservación y restauración del Patrimonio Cultural. https://www.researchgate.net/publication/323613615_Terminologia_basica_de_conser vacion_y_restauracion_del_Patrimonio_Cultural

Iglesias Llorente, F. M. (1885). Informe del director de los Archivos Nacionales. Año 1885. Revista del Archivo Nacional, año XXXI(1 - 12), 312 - 321.

Jaén García, L. F. (2017). Memorias Archivo Nacional: tomo 1 (1881-1916). No publicado.

Murillo Delgado, J. F. (1980). El Archivo Nacional: evolución histórica (1881-1979) (trabajo final para obtener el grado de Licenciado en historia). Universidad de Costa Rica, San José, CR.

Real Academia Española (2019). Cartivana. https://dle.rae.es/?id=7kbfBmG

Schweidler, M. (2006). The restoration of engravings, drawings, books, and other Works

on paper (R. Perkinson, trad.). The Getty Conservation Institute.

Taedium (s.f.). Caracteres externos e internos de los documentos. Recuperado el 30 de Julio del 2020 desde:

http://www.ub.edu/contrataedium/taediumcast/ordit/cultura_escrita/iden_estructures _formals/iframes/caracters.html

Vergara, J. (2002). Conservación y restauración de material cultural en archivos y bibliotecas. Valencia, España: Biblioteca Valenciana. 
Revista Herencia, Vol. 34 (1), enero-junio, 2021. 